Taku Komal. A case of conspicuous sexual difference in coloration in Stomatopods with notes of a male coloration approching that of the female (Ann. Zool. Japan, X, avril 1922, pp. 101-107)

\title{
NOTE SUR UNE ESPÈCE NOUVELLE D'HYDROÏDE DES CÔTES DE FRANCE (DYNAMENA DUBIA)
}

\author{
PAR \\ ARMAND BILLARD \\ Professeur à la Faculté des sciences de Poitiers.
}

Parmi les Hydrö̈des récoltés sur ma demande par le Dr CouLONGEAT, assistant de zoologie à mon laboratoire, j'ai trouvé une forme intéressante, que je considère comme nouvelle et que je propose d'appeler Dynamena dubia.

Les colonies, qui proviennent de la Pallice et sont fixées sur une petite Algue verte, ne dépassent pas $1 \mathrm{~cm}$. 5 ; leur hydrocaule est divisé en articles séparés par des lignes obliques et vue de face l'articulation se présente sous la forme de deux cònes se pénétrant par leur pointe (fig. 1, $A$ et $B$ ). Les hydrothèques sont strictement opposées et occupent la partie proximale de chaque article; sur l'une des faces elles se touchent, tandis que sur l'autre elles sont légèrement écartées. Leur fond du côté adcaulinaire est pourvu d'un processus plus ou moins développé.

Ce qui caractérise ces hydrothèques éest la forme de leur orifice qui est dirigé verticalement et figure un biseau ou un sifflet à bec court; ce large orifice est fermé par une grande valve operculaire abcaulinaire et en outre par une petite lame adcaulinaire relevée, continuant la paroi correspondante de l'hydrothèque, qui parait retroussée ; cette lame adcaulinaire est parfois difficile à mettre en évidence; les deux lames s'affrontent pour fermer l'orifice. Il n'y a pas de dents latérales et il semble que le large orifice se soit formé par le développement exagéré de l'échancrure abcaulinaire du genre Dynamena typique, tel que le définit Вrocн $(\mathbf{1})$, et par la réduction à zéro

(1) The Danish Ingolf Expedition, Hydroida (P. II, 1918, p. 114). 
de l'échancrure adcaulinaire, avec persistance de la lame adcaulinaire qui est alors rudimentaire et a pris un caractère spécial. Les hydranthes ne présentent pas de cul-de-sac abcaulinaire et c'est là une des raisons qui me fait placer cette forme

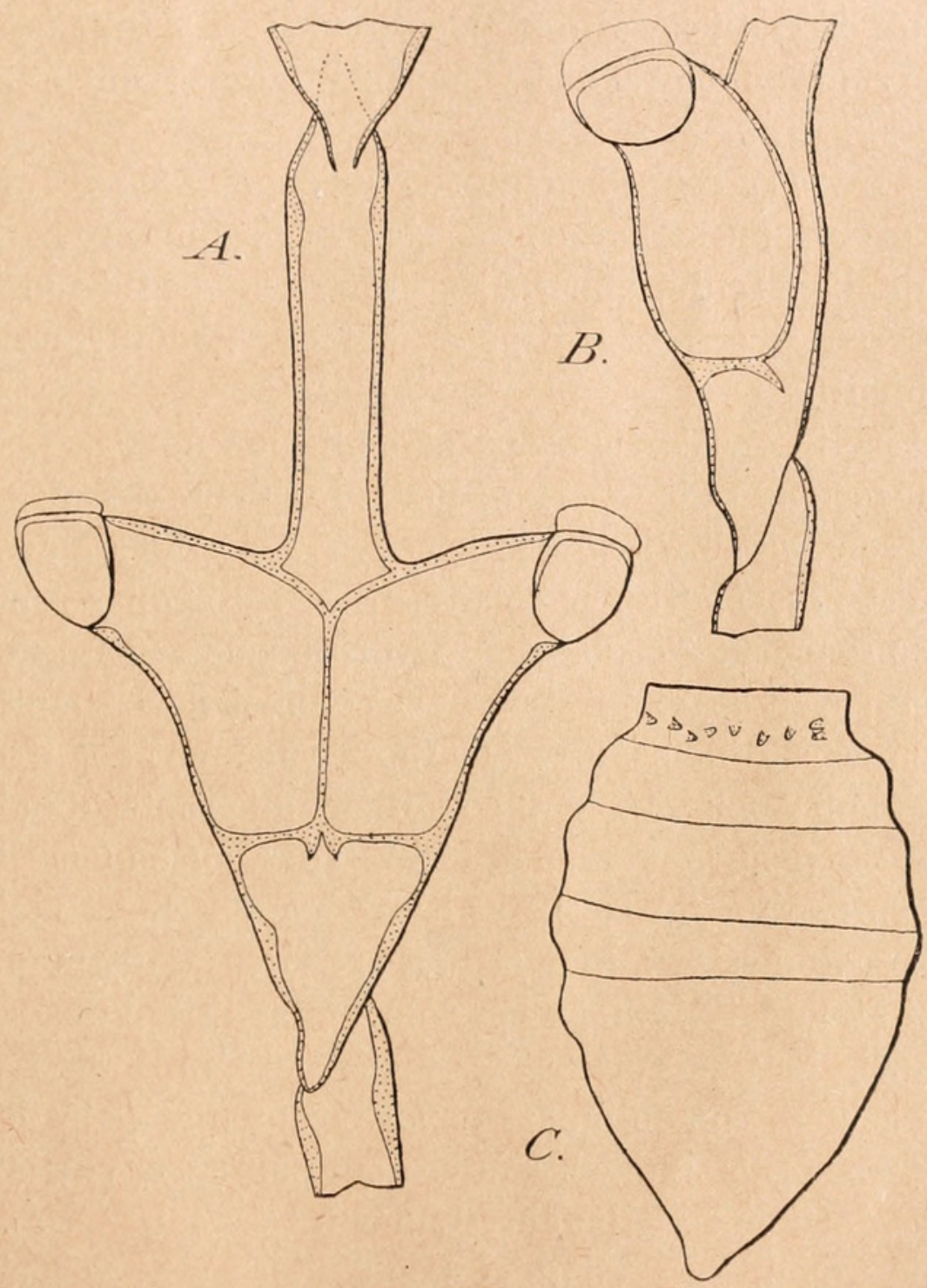

Fig. 1. - Dynamena dubia, n. sp.

$A$, partie d'hydrocaule, gr. $80 ; B$, hydrothèque montrant l'orifice vu de face, gr. $80 ; C$, gonothèque, gr. 49 .

dans le genre Dynamena, dont elle possède aussi les hydrothèques opposées. On pourrait à la rigueur la considérer comme le chef de file d'un nouveau genre, mais depuis ces dernières années les genres ont été tellement multipliés dans la famille des Sertularides que j'hésite à créer un nouveau genre; je préfère rattacher cette espèce au genre Dynamena, au moins provisoirement, malgré la forme et la disposition un 
peu différentes de l'opercule; sous ce rapport notre espèce établit le passage vers le genre Thuiaria, mais les hydranthes des Thuiaria possèdent un cul-de-sac abcaulinaire.

Les gonothèques sont ovales (fig. $1 C$ ), tronquées à leur extrémité distale, qui présente un col étroit à l'intérieur duquel se voient de petites saillies périsarcales; de plus ces gonothèques sont annelées, mais les annellations sont faiblement marquées.

Cette espèce se rapproche du Sertularia desmoides Torrey (1), qui appartient au mème groupe par les particularités de l'orifice et de l'opercule, mais la forme des hydrothèques est un peu différente; ces deux espèces ont des gonothèques qui se ressemblent.

J'ai récolté moi-mème des échantillons de cette espèce à Biarritz (rocher de la Vierge), mais les deux colonies que je possède sont plus petites $(3 \mathrm{~mm}$.) et la partie distale de chaque article est plus courte, les paires d'hydrothèques sont alors plus rapprochées; l'orifice des hydrothèques bien que moins visible apparait du même type. Les colonies ne portent pas de gonothèques. Le nanisme et le rapprochement des hydrothèques tiennent sans doute aux conditions différentes d habitat.

Enfin M. Dollfus a recueilli aussi des spécimens de cette espèce entre l'île de Groix et les Glénans, par $\mathbf{2 4}$ mètres de fond; les colonies ont pour la plupart $1 \mathrm{~cm}$. de hauteur, l'une pourtant atteignait $2 \mathrm{~cm} .5$; ces colonies montrent des rameaux stoloniques. Le plus souvent les articles, séparés aussi par des lignes d'articulation obliques, ne portent qu'une paire d'hydrothèques, mais parfois on en trouve deux paires; la partie distale de chaque article est allongée. L’une de ces colonies porte à la base une gonothèque complètement vide à paroi mince et onduleuse, largement ouverte à son extrémité distale, sans col, ni saillies périsarcales, mais il semble que cette gonothèque n'est pas complète.

Les dimensions des échantillons des différentes provenances sont comme on le voit très voisines, l'intervalle des hydrothèques est plus faible dans les colonies de Biarritz, comme je

(1) The Hydroida of the pacific coast of North America (Univ. California publications, Zoology, I, 1902, p. 63. pl. vir, fig. 70-72); et Nutrixg (C. C.) American Hydroids, P. II. The Sertularide (Smithson. Instit. U. S. Nat. Mus. Special Bull. 1904, p. 56, pl. III, fig. 1-3). 
l'ai fait remarquer et la partie soudée est plus grande dans les formes de Bretagne.

\begin{tabular}{|c|c|c|c|}
\hline \multirow{2}{*}{ Dimensions } & \multicolumn{3}{|c|}{ Échantillons } \\
\hline & $\begin{array}{c}\text { La Pallice } \\
\text { (type) }\end{array}$ & Biarritz & Bretagne \\
\hline $\begin{array}{l}\text { Longueur de la partie abeaulinaire des } \\
\text { hydrothèques } \\
\text { Longueur de la partie libre des hydro- } \\
\text { thèques. } \\
\text { Longueur de la partie soudée des hydro- } \\
\text { thèques. } \\
\text { Largeur des hydrothèques (à lorifice) } \\
\text { lntervalle entrelespaires d'hydrothèques } \\
\text { Largeur de l'hydrocaule } \\
\text { Longueur des gonothèques. } \\
\text { Largeur des }\end{array}$ & $\begin{array}{l}24.5-280 \mu \\
215-245 \% \\
26 \%-980 \mu \\
100-110 \% \\
44.5-760 \% \\
8.700 \% \\
1370-148 \% \mu \\
860-960 \%\end{array}$ & $\begin{array}{l}245-260 \mu \\
200-215 \mu \\
245-265 \mu \\
115-130 \mu \\
165-330 \mu \\
65-80 \mu\end{array}$ & $\begin{array}{l}245-260 \\
200-230 \\
300-345 \\
11-130 \\
430-345 \\
100-115 \\
1320 \mu \\
72 \% \%\end{array}$ \\
\hline
\end{tabular}

En terminant j'indiquerai que la forme signalée par moi comme une variété de Sertularia distans Lamx. et provenant de l'expédition du “ Travailleur » (1) doit être rapportée au Dynamena dubia, mais il s'agit d'une variété de taille plus grande (3 cm.5) présentant quelques ramifications. Les articles portent le plus souvent deux paires d'hydrothèques, mais certains n'en ont qu'une seule paire. C'est par suite d'une méprise que j'ai figuré deux dents latérales à l'orifice, qui est semblable à celui de notre type, dans les hydrothèques les mieux conservées; mais les lames operculaires sont absentes, une seule hydrothèque possédait encore dans une colonie la grande lame abcaulinaire. Les gonothèques manquent aussi à cet échantillon.

Les dimensions se rapprochent de celles des échantillons de Bretagne mais les dépassent, sauf pour la longueur de la partie soudée. Voici d'ailleurs ces dimensions pour cette variété :

(1) Expéditions scientifiquez du a Travailleur s et du a Talisman s. Hydroides (Paris, Masson, 1906, in-4, p. 191, fig. 11). 
Longueur de la partie abcaulinaire des hydrothèques. Longueur de la partie libre des hydrothèques. . $245-300 \mu$ Longueur de la partie soudée des hydrothèques.

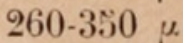
$315-330 \mu$ Largeur des hydrothèques (à l'orifice) $105-140 \mu$ Intervalle entre les paires d'hydrothèques. . . $410.775 \mu$ Largeur de l'hydrocaule.

$120-175 \mu$

\title{
COMPLÉMENT A LA DESCRIPTION DE CYCLOBOTHRIUM CHARCOTI MIHI
}

\author{
PAR
}

Robert-Ph. DOLLFUS

Un exemplaire du Trématode ectoparasite que nous avons décrit sous le nom de Cyclobothrium Charcoti (1) vient de nous être remis par notre ami Th. Mosov qui l'a recueilli à Monaco (21 octobre 1922) en examinant un couple de Meinertia oestroüdes Risso de la bouche de Box boops L.

Pour décrire $C$. Charcoti, nous n'avions à notre disposition qu'un seul spécimen; l'examen du spécimen trouvé par Th. Monod nous donne l'occasion de compléter notre descrip. tion première.

Nous avions indiqué que le lobe antérieur de ce parasite était petit, ovalaire, à peu près aussi long que large (environ $0,65 \mathrm{~mm}$.), il avait cette forme parce qu'il se trouvait à l'état de rétraction maximum; l'individu récolté par Movod montre en effet (fig. 1) un lobe antérieur long et étroit (environ $2 \mathrm{~mm}$. sur 0,5$)$; mais on remarquera qu'il n'est. pas à l'état d'extension complète (fig. 2 aussi peut-on prévoir que, complètement étendu, ce lobe antérieur pourrait mesurer les $\mathbf{2} / 3$ de la longueur totale du corps. Ce iobe est comparable à une sorte de trompe; il est pourvu d'une très forte musculature.

L'opacité du parenchyme et des vitellogènes rend les organes internes (en particulier l'intestin, l'ovaire et le receptaculum

(1) Cyclobothrium Charcoti, n. sp. Trémalode ectoparasite sur Meinertia astroides (Risso). Parasites recueillis pendant la croisière océanographique du "Pourquoi-pas?" sous le commandement du Dr J.-B. Снавcot, en 1914 (1 re note) (Bull. Soc. Zool France, XLVII, séance du 11 juillet 1922, pp. 287-296, fig. 1-3). 


\section{$2 \mathrm{BHL}$ Biodiversity Heritage Library}

Billard, Armand. 1922. "Note sur une espece nouvelle d'hydroïde des côtes de France (Dynamena dubia)." Bulletin de la Société zoologique de France 47, 344-348. https://doi.org/10.5962/bhl.part.3471.

View This Item Online: https://www.biodiversitylibrary.org/item/22091

DOI: https://doi.org/10.5962/bhl.part.3471

Permalink: https://www.biodiversitylibrary.org/partpdf/3471

\section{Holding Institution}

MBLWHOI Library

\section{Sponsored by}

MBLWHOI Library

\section{Copyright \& Reuse}

Copyright Status: NOT_IN_COPYRIGHT

This document was created from content at the Biodiversity Heritage Library, the world's largest open access digital library for biodiversity literature and archives. Visit BHL at https://www.biodiversitylibrary.org. 